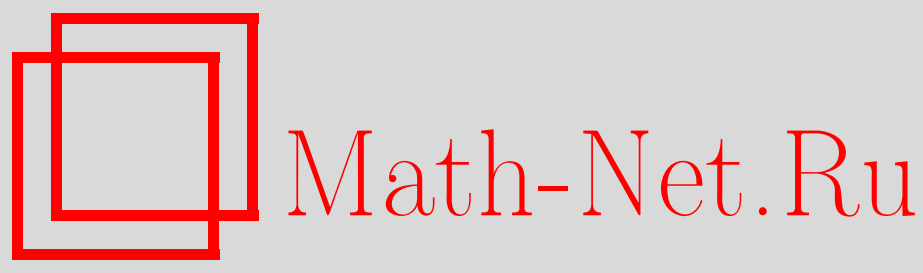

А. Ю. Хренников, Взгляд на группирование и антигруппирование с точки зрения классических сигналов, ТМФ, 2012, том 172, номер 1, 155-176

DOI: https://doi.org/10.4213/tmf6934

Использование Общероссийского математического портала Math-Net.Ru подразумевает, что вы прочитали и согласны с пользовательским соглашением http://www.mathnet.ru/rus/agreement

Параметры загрузки:

IP : 54.174 .149 .18

26 апреля 2023 г., 17:00:51

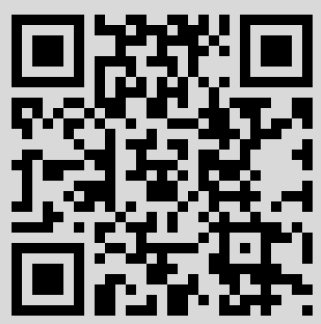




\title{
ВЗГЛЯД НА ГРУППИРОВАНИЕ И АНТИГРУППИРОВАНИЕ С ТОЧКИ ЗРЕНИЯ КЛАССИЧЕСКИХ СИГНАЛОВ
}

\begin{abstract}
Сходство квантовой механики с классической волновой механикой отмечалось в работах де Бройля, Шредингера, "позднего Эйнштейна", Лэмба, Ланде, Манделя, Маршалла, Сантоса, Бойера и многих других авторов. Представлена новая волновая модель квантовой механики - так называемая предквантовая классическая статистическая теория поля, в которой исследуется аналогия между некоторыми квантовыми явлениями и классической теорией случайных полей. Квантовые системы интерпретируются как символические представления таких полей (не только для фотонов, ср. подходы Ланде и Лэмба, но и для массивных частиц). Все квантовые средние и корреляции (включая составные системы в запутанных состояниях) можно представить как средние и корреляции классических случайных полей. Предквантовая классическая статистическая теория поля используется для получения группирования и антигруппирования в рамках классической теории сигналов. Заметим, что по крайней мере антигруппирование обычно рассматривается как существенно квантовое (неклассическое) явление.
\end{abstract}

Ключевые слова: квантовая механика как образ классической модели, классические случайные поля, квантовые корреляции, классические корреляции, группирование, антигруппирование.

\section{1. ВВЕДЕНИЕ}

Среди специалистов по основаниям квантовой механики (KM) общепринята точка зрения, что квантовые корреляции невозможно свести к корреляциям классических случайных полей (см., например, работы [1], [2]). Однако в то же время были разработаны различные "предквантовые модели", включая модели, основанные на классических полях (см., например, работы [3]-[26]). Эти модели воспроизводят некоторые фундаментальные характеристики КМ. Каждая модель обладает своими достоинствами и недостатками. Особенно привлекателен классический полевой подход

*International Center for Mathematical Modelling in Physics and Cognitive Sciences, Linnaeus University, Växjö, Sweden. E-mail: andrei.khrennikov@lnu.se 
к КМ. Этот подход основан на идеях Планка, Ми, Шредингера, де Бройля и “позднего Эйнштейна"1) (см., например, работу [2]). Основными моделями классического полевого подхода к микроявлениям являются квазиклассический подход к квантовой электродинамике и тесно связанная с ним стохастическая электродинамика (СЭД) [3]-[11]. Недавно была предложена новая модель волнового типа - предквантовая классическая статистическая теория поля (ПКСТП) [28]-[34]. Это чисто полевая модель, т. е. частицы полностью исключаются из рассмотрения (ср. работы Ми, Эйнштейна, Шредингера). Не только фотоны, но и все квантовые системы, например электроны, описываются классическими случайными полями. Квантовые измерения интерпретируются как измерения классических случайных сигналов. В некотором смысле ПКСТП является моделью со скрытыми переменными. На современном уровне развития техники мы не способны отслеживать поведение предквантовых сигналов, поскольку они флуктуируют на предквантовой временно́й шкале, которая, существенно меньше временно́й шкалы сегодняшних измерений [35]. Однако можно ожидать, что в будущем удастся (как надеялся Эйнштейн) приблизиться к предквантовой временно́й шкале и, забыв о квантовом формализме (который дает лишь очень грубое описание микроявлений), работать в рамках теории классических сигналов ${ }^{2)}$.

Стоит еще раз подчеркнуть, что сигналы являются случайными. Таким образом, неясно, можно ли рассматривать ПКСТП как реализацию мечты Эйнштейна о восстановлении детерминизма. Одной из основных отличительных черт ПКСТП является предположение о том, что случайность предквантовых полей, соответствующих квантовым системам, фундаментальным образом связана со случайным фоновым полем (“флуктуациями вакуума"). Случайное фоновое поле играет решающую роль и в других классических полевых моделях, особенно в СЭД ${ }^{3)}$. В рамках ПКСТП фоновое поле является неприводимой компонентой случайных полей, представляющих квантовые системы. В отличие от, например, СЭД в ПКСТП мы не можем выделить "внутреннее поле", скажем, электрона, отфильтровав фоновое поле. Такого собственного поля квантовой системы не существует. Его невозможно выделить из флуктуаций вакуума. "Фильтрацию” можно осуществить только на уровне статистики измерений (см. раздел 4 настоящей работы, а также [38]-[40]). Эта процедура описывается с помощью КМ. Таким образом, вероятностные величины в КМ не совпадают с классическими. Квантовые получаются путем перенормировки классических (см. ниже формулу (11)), поэтому квантовые и классические модели связаны весьма нетривиальным образом ${ }^{4}$. При этом такая связь возможна только в присутствии случайного фонового поля. Это поле и является источником дополнительных

1) В 1905 г. Эйнштейн изобрел квант света. Однако начиная с 1920-х гг. он начал работать над чисто классической волновой моделью физической реальности [27].

2) Можно действовать в обратном направлении, т. е. создать квантовоподобное представление классической теории сигналов [36]; в частности, грубое описание классических оптических явлений индуцирует (макроскопическую) модель типа квантовой [37].

3) Планк был первым, кто указал (в 1908 г. он спорил об этом с "ранним Эйнштейном"), что, учитывая случайное фоновое поле, можно избежать квантовых скачков.

4) Хотя мы работаем в рамках КM, а не квантовой теории поля, перенормировка, связывающая КМ и ПКСТП, напоминает процедуру перенормировки вакуума в квантовой теории поля. Для некоторых классических величин (заданных в виде квадратичных форм от классических полей, соответствующих неядерным операторам), чтобы получить квантовую величину, нужно из соответствующих классических величин вычесть бесконечность. 
корреляций. В принципе можно рассматривать фоновое поле как источник "нелокальных корреляций”. Однако это поле является классическим, а потому представляет собой классическую волновую “нелокальность". Присутствие случайного фонового поля (в комбинации со связью между квантовыми и классическими величинами в духе перенормировки) позволяет создать классическую вероятностную модель антигруппирования (в принципе возможность классического описания группирования не выглядит удивительной). С учетом упомянутых свойств ПКСТП можно избежать проблем, связанных с существованием доказательств невозможности классического вероятностного описания антигруппирования; так, в работе [41] приведено одно из таких доказательств, представленное в строгих математических рамках, основанное на субпуассоновой статистике, а также неравенстве Коши-Буняковского.

В сжатом виде идеи ПКСТП состоят в следующем:

1) квантовые системы, несмотря на нетривиалъные квантовые явления, могут отображаться на классические стохастические системы;

2) квантовые явления более близки к классическим, чем это обычно принято.

Примеры отображений с приведенными выше свойствами хорошо известны: это $Q$-представление для линейных бозонных систем и так называемое положительное $P$-представление для нелинейной оптики. Для квантовых состояний $Q$-функция электромагнитного поля положительна, что не противоречит нарушению неравенства Белла, начиная с эксперимента Аспекта. Более общим образом, квантовый томографический подход (см., например, работы [17]-[20]) позволяет представить все квантовые средние и корреляции, используя классические распределения вероятностей. Хотя корреляции ЭПР можно представить как корреляции классических случайных переменных, например в квантовом томографическом подходе и в ПКСТП, нарушение неравенства Белла не удивительно: некоторые условия, использованные Беллом при выводе его "теорем", нарушаются"). В частности, в ПКСТП нарушается спектральный постулат: "предквантовые переменные" (задаваемые квадратичными формами классических полей) не обладают той же областью значений, что и их квантовые аналоги. Условие, что наблюдаемые принимают значения \pm 1 , играет ключевую роль в исходном неравенстве Белла для корреляций [44]. Неравенство Клаузера-Хорна-Шимони-Хольта основано на более слабом ограничении на область значений наблюдаемых: они должны принимать значения на отрезке $[-1,1]$. Однако даже последнее условие нарушается в ПКСТП, поэтому основная нерешенная проблема ПКСТП - это создание новой теории измерений, которая воспроизводила бы результаты измерений в КМ из взаимодействий классических полей. Первые шаги в этом направлении уже сделаны: см. работу [33] и в особенности работу [45].

Настоящую работу мы начнем с краткого представления ПКСТП. В основном это теория классических случайных полей [28]-[32], [38]-[40]. Однако, чтобы связать ПКСТП с КМ, мы должны переписать ее, используя язык операторов и следов. В разделе 4 мы представляем квантовые корреляции как корреляции квадратичных форм классических случайных сигналов. В разделе 5 обсуждается роль фаз в ПКСТП и КМ. Посредством формализма ПКСТП волновая функция $\Psi$ составной

5) Анализ роли пространственно-временной зависимости корреляций можно найти, например, в работе [42] (см. также монографию [43]). 
квантовой системы $S=\left(S_{1}, S_{2}\right)$ определяет ковариационный оператор $D_{\Psi}$ соответствующего предквантового случайного поля. Хорошо известно, что в КМ вектор чистого состояния определяется с точностью до фазового множителя. Однако два вектора, представляющие одно и то же чистое квантовое состояние и имеющие различные фазы (с точностью до $2 \pi$ ), определяют два различных предквантовых поля. Они дают одинаковые корреляции для квадратичных форм, т. е. воспроизводят одинаковые квантовые корреляции. В п. 6.1 мы расссматриваем классические случайные бисигналы (бисигналы, инвариантные относительно перестановки компонент), которые воспроизводят бозонные квантовые корреляции. Если бисигнал инвариантен относительно перестановки компонент, дополненной сдвигом на $\pi$ относительной фазы между компонентами (см. п. 6.2), то он воспроизводит фермионные корреляции. Раздел 8 посвящен представлению некоторых квантовых каналов как каналов классических сигналов (ср. с работами [36], [46]). В разделе 10 мы исследуем (анти)группирование случайных полей без спина, взаимодействующих со светоделителями, а в разделе 12 представлено (анти)группирование классических случайных полей со спином $1 / 2$.

Реальное физическое пространство состояний классических (а также квантовых) сигналов - это пространство $L_{2}$. Данное пространство бесконечномерно, и теория случайных переменных в этом пространстве (случайных полей) сложна с математической точки зрения, поэтому читатель может в принципе рассматривать конечномерное гильбертово пространство и случайные переменные, принимающие значения в этом пространстве. В ПКСТП удобно оперировать гауссовыми случайными полями. В конечномерном случае они представляют собой просто (комплекснозначные) гауссовы случайные переменные.

Читатель, не слишком интересующийся проблемой полноты КМ и существованием скрытых переменных (и, возможно, оснований КМ в целом), может рассматривать настоящую работу как вклад в моделирование квантовых явлений в классических вероятностных терминах (ср. с работами [47]-[49]).

\section{2. ПРЕДСТАВЛЕНИЕ КЛАССИЧЕСКОГО ПОЛЯ ДЛЯ НЕСОСТАВНЫХ КВАНТОВЫХ СИСТЕМ}

2.1. Классические поля как скрытые переменные. С математической точки зрения классические поля суть функции $\phi: \mathbb{R}^{3} \rightarrow \mathbb{C}$ (или, более общо, функции со значениями в $\left.\mathbb{C}^{k}\right)$, которые являются квадратично-интегрируемыми, т. е. элементами пространства $L_{2}\left(\mathbb{R}^{3}\right)$. Случайное поле (в фиксированный момент времени) это функция $\phi(x, \omega)$, где $\omega$ - случайный параметр. Таким образом, для каждого $\omega_{0}$ мы получаем классическое поле, $x \mapsto \phi\left(x, \omega_{0}\right)$. Согласно ПКСТП каждая квантовая система - это символическое представление классического случайного поля, предквантовое поле. Пространство состояний нашей предквантовой модели то же, что и в случае теории классических сигналов: $H=L_{2}\left(\mathbb{R}^{3}\right)$. Действуя линейным функционалом $y$ на случайный вектор $\phi$, получаем скалярную случайную переменную. Когда $y$ пробегает все пространство $L_{2}$, получаем семейство скалярных случайных переменных

$$
\omega \mapsto \xi_{y}(\omega) \equiv \int y(x) \overline{\phi(x, \omega)} d x, \quad y \in L_{2}\left(\mathbb{R}^{3}\right)
$$


Напомним, что ковариационный оператор $D$ случайного поля (с нулевым средним) $\phi \equiv \phi(x, \omega)$ определяется своей билинейной формой: $\langle D u, v\rangle=E\langle u, \phi\rangle\langle\phi, v\rangle$, $u, v \in H$, где $E$ - классическое математическое ожидание (среднее значение). Далее мы будем дополнительно предполагать, что все предквантовые поля являются гауссовыми и имеют нулевое среднее: $E\langle y, \phi\rangle=0$ для всех $y \in H$. При этих условиях ковариационный оператор определяет поле единственным образом.

\section{2. Интерпретация волновой функциии с помощью ковариационного} оператора. В нашей модели волновая функция $\Psi$ из формализма KM задает предквантовое случайное поле: $\phi \equiv \phi_{\Psi}$. Терминологии КМ “квантовая система в состоянии $\Psi$ " отвечает терминология ПКСТП "случайное поле". В ПКСТП $\Psi$-функция определяет ковариационный оператор предквантового случайного поля. Для простоты рассмотрим случай одиночной, т. е. несоставной, системы, например фотон или электрон. В такой ситуации нормировка (на дисперсию) ковариационного оператора $D$ предквантового поля имеет вид ортогонального проектора на вектор $\Psi$ (оператора плотности, отвечающего этому чистому состоянию): $\rho_{\Psi}=|\Psi\rangle \otimes\langle\Psi|$, т. е. $\rho_{\Psi} u=\langle u, \Psi\rangle \Psi, u \in H$. Ковариационный оператор предквантового поля имеет вид $D=\sigma^{2}(\phi) \rho_{\Psi}$, где $\sigma^{2}(\phi)=E\|\phi\|^{2}(\omega)=\operatorname{Tr} D$ - дисперсия предквантового случайного поля $\phi$, распределенного нормально с нулевым средним и ковариационным оператором $D$. Чтобы найти ковариационый оператор $D$, соответствующий оператору плотности $\rho_{\Psi}$, нужно найти шкалу флуктуаций предквантового поля, заданную дисперсией $\sigma^{2}(\phi)$.

Предквантовое случайное поле $\phi(x, \omega)$ не нормировано в пространстве $L_{2}\left(\mathbb{R}^{3}\right)$. Его интенсивность (норма)

$$
I(\phi(\omega))=\|\phi\|^{2}(\omega) \equiv \int_{\mathbb{R}^{3}}|\phi(x, \omega)|^{2} d x
$$

флуктуирует в зависимости от случайного параметра $\omega$. Мы интересуемся средним значением этой величины $\langle I\rangle \equiv E I(\phi)$. С точки зрения теории вероятностей это не что иное, как дисперсия случайного поля: $\langle I\rangle=\sigma^{2}(\phi)$, поэтому, чтобы найти шкалу предквантовых флуктуаций, нам нужно найти среднюю интенсивность этого поля. Поскольку мы не можем отслеживать флуктуации предквантовых полей, соответствующих квантовым системам, невозможно (на настоящий момент) найти упомянутую шкалу. Далее мы имеем дело (что типично для теории вероятностей) с нормированными предквантовыми полями, т. е. выполняем преобразование $\phi \rightarrow \phi / \sigma$.

\section{3. Квантовые наблюдаемые как квадратичные формы предквантово-} го поля. Самосопряженный оператор $\hat{A}$ рассматривается как символическое представление переменной ПКСТП: $\phi \mapsto f_{A}(\phi)=\langle\hat{A} \phi, \phi\rangle$. Заметим, что $f_{A}$ можно рассматривать как функцию на фазовом пространстве классических полей: $f_{A} \equiv$ $f_{A}(q, p)$, при этом $\phi(x)=q(x)+i p(x), q, p \in L_{2}\left(\mathbb{R}^{3} ; \mathbb{R}\right)$, где $L_{2}\left(\mathbb{R}^{3}, \mathbb{R}\right)$ - пространство квадратично-интегрируемых полей, действующих из $\mathbb{R}^{3}$ в $\mathbb{R}$.

Рассмотрим квантовое среднее $\left\langle f_{A}\right\rangle_{\mathrm{QM}}=\langle\hat{A} \psi, \psi\rangle$ и классическое среднее $\left\langle f_{A}\right\rangle_{\mathrm{CL}}=$ $E f_{A}(\phi)$. Они совпадают с точностью до скейлингового множителя:

$$
\left\langle f_{A}\right\rangle_{\mathrm{CL}}=\operatorname{Tr} D\left\langle f_{A}\right\rangle_{\mathrm{QM}}
$$


Для нормированных предквантовых полей они в точности равны друг другу. Такая связь между классическими и квантовыми средними была установлена в работах Баха [15], [16], которые стимулировали исследования [36] по квантовоподобному подходу к теории классического сигнала. Затем работы Баха были практически забыты. Одна из причин забвения классического представления Баха для квантовых средних заключалась в том, что ему удалось рассмотреть только несоставные квантовые системы. Квантовые корреляции, например, для запутанных систем, не были сведены к классическим корреляциям. Это было сделано в работах автора [38], [39], который не знал о работах Баха и независимым образом открыл соотношение (1).

\section{3. ОПЕРАТОРНОЕ ПРЕДСТАВЛЕНИЕ ЧИСТОГО СОСТОЯНИЯ СОСТАВНОЙ СИСТЕМЫ}

В данном разделе излагается новое математическое представление стандартного формализма KM. Использование получающегося в результате нового математического представления основных выражений КМ играет важную роль при построении связи между квантовыми корреляциями и корреляциями классических случайных полей, представляющих составные квантовые системы. Мы даем операторную интерпретацию чистых состояний составной квантовой системы $S=\left(S_{1}, S_{2}\right)$. В каноническом математическом формализме КМ чистые состояния представляются (нормированными) векторами, которые принадлежат тензорному произведению $H_{1} \otimes H_{2}$ пространств состояний подсистем $S_{1}$ и $S_{2}$. В нашем подходе они представляются линейными операторами, действующими из $H_{2}$ в $H_{1}$.

Пусть $\left\{e_{j}\right\}$ и $\left\{f_{j}\right\}$ - ортонормированные базисы в $H_{1}$ и $H_{2}$ соответственно. Возьмем $\Psi \in H_{1} \otimes H_{2}$,

$$
\Psi=\sum_{i, j} \Psi_{i j} e_{i} \otimes f_{j}, \quad\|\Psi\|^{2}=\sum_{i, j}\left|\Psi_{i j}\right|^{2}=1, \quad \Psi_{i j} \in \mathbb{C} .
$$

Для вектора $\phi \in H_{2}$ положим

$$
\widehat{\Psi} \phi=\sum_{i, j} \Psi_{i j}\left\langle\phi, \bar{f}_{j}\right\rangle e_{i}
$$

(использование комбинации векторов $\bar{f}_{j}$ и $e_{i}$ важно для получения формул, которые согласуются с векторным представлением чистых состояний составных систем в канонической математике в рамках KM). Здесь $\widehat{\Psi}: H_{2} \rightarrow H_{1}$ - операторное представление чистого состояния $\Psi \in H_{1} \otimes H_{2}$ составной квантовой системы $S=\left(S_{1}, S_{2}\right)$.

Теперь рассмотрим очень специальный, но в то же время очень важный случай: будем считать, что $H_{i}=L_{2}\left(\mathbb{R}^{n_{i}}\right)$ - пространство квадратично-интегрируемых комплекснозначных функций, $i=1,2$. При этом наше определение дает следующее представление:

$$
\widehat{\Psi} \phi(x)=\int \Psi(x, y) \phi(y) d y
$$

Далее мы действуем формально и используем вещественный базис $\left\{e_{x} \otimes f_{y}\right\}$, где $e_{x}(t)=\delta(t-x)$ и $f_{y}(s)=\delta(s-y)$. 


\section{1. Операция комплексного сопряжения в пространстве операторов.} Рассмотрим линейный оператор $\hat{A}: H_{2} \rightarrow H_{1}$. Определим комплексно-сопряженный onepamop $\hat{\bar{A}}$ с помощью его билинейной формы:

$$
\langle\hat{\bar{A}} u, v\rangle=\langle\bar{v}, \hat{A} \bar{u}\rangle .
$$

Для вещественного базиса имеем $\left\langle\hat{\bar{A}} e_{i}, e_{j}\right\rangle=\left\langle e_{j}, \hat{A} e_{i}\right\rangle=\overline{\left\langle\hat{A} e_{i}, e_{j}\right\rangle}$, поэтому матричные элементы комплексно-сопряженного оператора $\hat{\bar{A}}$ являются сопряженными матричным элементам оператора $\hat{A}$. Оператор называется вещественным, если $\hat{\bar{A}}=\hat{A}$; в вещественном базисе матричные элементы вещественного оператора являются вещественными числами. Для вещественных операторов наше рассмотрение существенно упрощается, однако наш формализм нельзя ограничить вещественными операторами. Например, оператор координаты и все гамильтонианы являются вещественными, а оператор импульса - нет. Более того, в общем случае оператор $\widehat{\Psi}$, соответствующий чистому состоянию $\Psi$, не является вещественным. Из определения (3) следует, что

$$
\left\langle\hat{A}^{*} \bar{v}, \bar{u}\right\rangle=\langle\hat{\bar{A}} u, v\rangle
$$

Поскольку $\overline{\hat{A}^{*}}=(\hat{\bar{A}})^{*}$, имеем

$$
\langle\hat{A} \bar{v}, \bar{u}\rangle=\left\langle(\hat{\bar{A}})^{*} u, v\right\rangle \text {. }
$$

Рассмотрим квадратичную форму комплексно-сопряженого оператора $\hat{\bar{A}}$ для самосопряженного оператора $\hat{A}$ :

$$
f_{\bar{A}}(\phi)=\langle\hat{\bar{A}} \phi, \phi\rangle=\langle\bar{\phi}, \hat{A} \bar{\phi}\rangle=f_{A}(\bar{\phi}) .
$$

Рассмотрим группу $\{e, *\}$, где $*$ - операция комплексного сопряжения в комплексном гильбертовом пространстве. Она индуцирует действие в пространстве вещественнозначных функций, заданных на комплексном гильбертовом пространстве, по правилу $f \rightarrow \bar{f}$, где $\bar{f}(\phi)=f(\bar{\phi})$ (мы надеемся, что символ $\bar{f}$ не будет вводить в заблуждение: рассматриваются только вещественнозначные функции, поэтому его нельзя спутать с операцией комплексного сопряжения в области значений).

\section{2. Основное операторное равенство для самосопряженных операто-} ров. Пусть $\Psi \in H_{1} \otimes H_{2}$. Тогда для любой пары самосопряженных (ограниченных) операторов $\hat{A}_{i}: H_{i} \rightarrow H_{i}, i=1,2$,

$$
\operatorname{Tr} \widehat{\Psi} \hat{\bar{A}}_{2} \widehat{\Psi}^{*} \hat{A}_{1}=\left\langle\hat{A}_{1} \otimes \hat{A}_{2}\right\rangle_{\Psi} \equiv\left\langle\hat{A}_{1} \otimes \hat{A}_{2} \Psi, \Psi\right\rangle
$$

Правая часть этого равенства дает квантовое среднее наблюдаемой $\hat{A}_{1} \otimes \hat{A}_{2}$, что основано на канонической векторной интерпретации чистого состояния $\Psi$ составной системы $S=\left(S_{1}, S_{2}\right)$. Левая часть равенства задает операторное представление для данного квантового среднего. Мы будем использовать это операторное представление в процессе перехода от нашей предквантовой модели к KM (напомним, что в данном разделе мы лишь исследуем новую математическую структуру КМ, а именно операторное представление чистых состояний). 
3.3. Операторное представление редуцированных операторов плотности. Рассмотрим оператор плотности $\rho \equiv \rho_{\Psi}=|\Psi\rangle \otimes\langle\Psi|$ и соответствующие редуцированные операторы плотности $\rho_{i} \equiv \operatorname{Tr}_{H_{i}} \rho_{\Psi}, i=1,2$. Выполняются равенства

$$
\rho_{1}=\widehat{\Psi} \widehat{\Psi}^{*}, \quad \bar{\rho}_{2}=\widehat{\Psi}^{*} \widehat{\Psi} .
$$

Появление комплексного сопряжения в последнем равенстве играет важную роль в самосогласовании связи квантовых и предквантовых корреляций. Заметим, что для любого самосопряженного оператора $\hat{A}$ имеет место равенство

$$
\operatorname{Tr} \bar{\rho} \hat{\bar{A}}=\operatorname{Tr} \rho \hat{A} .
$$

\section{4. ПРЕДСТАВЛЕНИЕ КЛАССИЧЕСКОГО СЛУЧАЙНОГО ПОЛЯ ДЛЯ КВАНТОВЫХ КОРРЕЛЯЦИЙ}

Рассмотрим составную квантовую систему $S=\left(S_{1}, S_{2}\right)$. Комплексное гильбертово пространство $H_{i}$ есть пространство состояний подсистемы $S_{i}, i=1,2$. Пусть $\phi_{1}(\omega)$ и $\phi_{2}(\omega)$ - гауссовы случайные поля (с нулевыми средними) в гильбертовых пространствах $H_{1}$ и $H_{2}$ соответственно. Рассмотрим декартово произведение $H_{1} \times H_{2}$, а также векторное гауссово случайное поле $\phi(\omega)=\left(\phi_{1}(\omega), \phi_{2}(\omega)\right) \in H_{1} \times H_{2}$. В данном случае его ковариационный оператор имеет блочную структуру:

$$
D=\left(\begin{array}{ll}
D_{11} & D_{12} \\
D_{21} & D_{22}
\end{array}\right),
$$

где $D_{i i}: H_{i} \rightarrow H_{i}$ и $D_{i j}: H_{j} \rightarrow H_{i}$. Ковариационный оператор самосопряжен, поэтому $D_{i i}^{*}=D_{i i}$ и $D_{12}^{*}=D_{21}$. Диагональные блоки $D_{i i}$ описывают внутренние корреляции в подсистемах, блок $D_{12}$ описывает кросс-корреляции между подсистемами. Здесь по определению

$$
\left\langle D_{i j} u_{j}, v_{i}\right\rangle=E\left\langle u_{j}, \phi_{j}(\omega)\right\rangle\left\langle\phi_{i}(\omega), v_{i}\right\rangle, \quad u_{i} \in H_{i}, \quad v_{j} \in H_{j} .
$$

Положим

$$
\begin{aligned}
\left\langle f_{A_{1}}, f_{A_{2}}\right\rangle & \equiv E f_{A_{1}} \bar{f}_{A_{2}}=\int_{H_{1} \times H_{2}} f_{A_{1}}\left(\phi_{1}\right) \bar{f}_{A_{2}}\left(\phi_{2}\right) d \mu\left(\phi_{1}, \phi_{2}\right)= \\
& =\int_{H_{1} \times H_{2}} f_{A_{1}}\left(\phi_{1}\right) f_{A_{2}}\left(\bar{\phi}_{2}\right) d \mu\left(\phi_{1}, \phi_{2}\right),
\end{aligned}
$$

где $\mu$ - распределение вероятностей случайного векторного поля $\phi$. Положим также

$$
\operatorname{cov}\left(f_{A_{1}}, f_{A_{2}}\right)=\left\langle f_{A_{1}}, f_{A_{2}}\right\rangle-\left\langle f_{A_{1}}\right\rangle\left\langle\bar{f}_{A_{2}}\right\rangle \text {. }
$$

Можно доказать (вычисляя гауссовы интегралы), что для любого гауссова случайного поля $\phi(\omega)$, принимающего значения в $H_{1} \times H_{2}$, с нулевым средним и ковариационной матрицей $D$ такой, что внедиагональный блок имеет вид $D_{12}=\widehat{\Psi}$, справедливо равенство

$$
\operatorname{cov}\left(f_{A_{1}}, f_{A_{2}}\right)=\left(\hat{A}_{1} \otimes \hat{A}_{2} \Psi, \Psi\right) \equiv\left\langle\hat{A}_{1} \otimes \hat{A}_{2}\right\rangle_{\Psi}
$$

Это равенство устанавливает связь между квантовыми и классическими корреляциями. 


\section{1. Диагональные блоки ковариационного оператора предквантового} случайного поля. Операторы $D_{i i}$ отвечают за средние значения функционалов, зависящих только от одной из компонент случайного векторного поля $\phi(\omega)$. В частности, $E f_{A_{1}}\left(\phi_{1}(\omega)\right)=\operatorname{Tr} D_{11} \hat{A}_{1}$ и $E \bar{f}_{A_{2}}\left(\phi_{2}(\omega)\right)=\operatorname{Tr} D_{22} \hat{\bar{A}}_{2}$. Построим случайное поле такое, что эти "маргинальные средние" будут соответствовать средним, полученным в рамках KM. Для последних имеем

$$
\left\langle\hat{A}_{1}\right\rangle_{\Psi}=\left(\hat{A}_{1} \otimes I \Psi, \Psi\right)=\operatorname{Tr} \rho_{1} \hat{A}_{1}, \quad\left\langle\hat{A}_{2}\right\rangle_{\Psi}=\left(I \otimes \hat{A}_{2} \Psi, \Psi\right)=\operatorname{Tr} \rho_{2} \hat{A}_{2},
$$

где $I$ - единичный оператор. Используя первое равенство из (5), первое среднее можно записать как $\left\langle\hat{A}_{1}\right\rangle_{\Psi}=\operatorname{Tr}\left(\widehat{\Psi} \widehat{\Psi}^{*}\right) \hat{A}_{1}$. С учетом равенства (6) второе среднее можно представить как $\left\langle\hat{A}_{2}\right\rangle_{\Psi}=\operatorname{Tr} \bar{\rho}_{2} \hat{\bar{A}}_{2}$ и, наконец, используя второе равенство в $(5)$, получаем $\left\langle\hat{A}_{2}\right\rangle_{\Psi}=\operatorname{Tr}\left(\widehat{\Psi}^{*} \widehat{\Psi}\right) \hat{\bar{A}}_{2}$. Таким образом, было бы естественно взять ковариационный оператор

$$
D=\left(\begin{array}{cc}
\widehat{\Psi} \widehat{\Psi}^{*} & \widehat{\Psi} \\
\widehat{\Psi}^{*} & \widehat{\Psi}^{*} \widehat{\Psi}
\end{array}\right) .
$$

Его внедиагональный блок воспроизводит правильные квантовые корреляции между системами $S_{1}$ и $S_{2}$, а его диагональные блоки дают правильные квантовые средние для системы $S_{1}$ и системы $S_{2}$.

Однако в общем случае (т. е. для произвольного чистого состояния $\Psi$ ) этот оператор не является положительно определенным. Рассмотрим модифицикацию этого оператора, которая будет положительно определена, и при этом квантовые и классические средние будут связаны простым правилом. Таким образом, из квантовых средних можно будет легко найти классические средние и наоборот. Для любого нормированного вектора $\Psi \in H_{1} \otimes H_{2}$ и достаточно большого $\epsilon>0$ оператор

$$
D \equiv D_{\Psi}=\left(\begin{array}{cc}
\widehat{\Psi} \widehat{\Psi}^{*}+\epsilon I & \widehat{\Psi} \\
\widehat{\Psi}^{*} & \widehat{\Psi}^{*} \widehat{\Psi}+\epsilon I
\end{array}\right)
$$

является положительно определенным [38]-[40]. Модифицикацию диагональных членов можно интерпретировать как учет фонового поля, представляющего собой случайное поле типа белого шума, т. е. гауссово поле с нулевым средним и ковариационным оператором, равным $\epsilon I$. Невозможно построить предквантовое случайное поле, соответствующее квантовым корреляциям, без учета наличия фонового поля (“флуктуаций вакуума"). Это очень важный момент нашей теории. С математической точки зрения ситуация является даже более замысловатой.

Невозможно выделить "внутреннее поле" квантовой системы, например электрона, из случайного фонового поля. Такое "внутреннее электронное поле" не существует, оно имеет смысл только в комбинации с фоновым полем. Это отражает реально существующую ситуацию, т. е. то, что имеет место в природе (без всякого отношения к нашим измерениям). В то же время ясно, что в теории измерений следует исключить вклад вакуумных флуктуаций. Это было сделано в канонической КМ (следуя Бору, рассмотрим КМ как теорию измерений). Обсудим этот важнейший момент более подробно.

Для гауссовой меры с ковариационным оператором (8) имеем

$$
\left\langle\hat{A}_{1}\right\rangle_{\Psi}=E f_{A_{1}}\left(\phi_{1}(\omega)\right)-\epsilon \operatorname{Tr} \hat{A}_{1}, \quad\left\langle\hat{A}_{2}\right\rangle_{\Psi}=E f_{A_{2}}\left(\phi_{2}(\omega)\right)-\epsilon \operatorname{Tr} \hat{A}_{2} .
$$


Эти соотношения для средних и соотношение (7) для корреляции обеспечивают связь между ПКСТП и КМ. Квантовые статистические величины можно получить из соответствующих величин для классического случайного поля. Можно было бы сказать, что "неприводимые квантовые случайности" редуцируются к случайностям классических предквантовых полей (ср. с монографией [50]). Однако ситуация является более сложной.

Из равенств (9) следует, что квантовые средние получены как перенормировки типа сдвига, примененные к средним по классическим случайным полям. Сдвиг соответствует устранению вклада фонового поля. Таким образом, квантовые средние не являются просто классическими средними, поэтому в некотором смысле фон Нейман [50] был прав. Квантовые средние не представляются в виде

$$
\left\langle\hat{A}_{2}\right\rangle_{\Psi}=E g_{A}(\phi(\omega)),
$$

где $\phi_{\Psi} \equiv \phi(\omega)$ - предквантовое случайное поле, соответствующее квантовому состоянию $\Psi$, а $g_{A}(\phi)$ - функционал предквантового поля.

Игнорируя математические усложнения, обусловленные бесконечной размерностью пространства, можно записать равенства (9) как

$$
\left\langle\hat{A}_{1}\right\rangle_{\Psi}=E f_{A_{1}}\left(\phi_{1}(\omega)\right)-\epsilon E f_{A_{1}}(\eta(\omega)), \quad\left\langle\hat{A}_{2}\right\rangle_{\Psi}=E f_{A_{2}}\left(\phi_{2}(\omega)\right)-\epsilon E f_{A_{2}}(\eta(\omega)),
$$

где $\eta$ - белый шум: гауссова случайная переменная с нулевым средним и единичной ковариационной матрицей. Хотя мы не получили представления (10), представление (11) можно интерпретировать как редукцию квантовой случайности к случайности классического ансамбля, поэтому в некотором смысле Эйнштейн [27] тоже был прав. В рамках ПКСТП позиции фон Неймана и Эйнштейна мирно объединяются. Однако автор не уверен, что фон Нейман или Эйнштейн были бы довольны таким мирным соглашением между копенгагенским лагерем и лагерем приверженцев классического подхода. Фон Нейман определенно был убежден, что загадка квантовых случайностей не сводится к преобразованию типа сдвига (11), а Эйнштейн не интересовался редукцией квантовых эффектов к случайному фону. Во всяком случае он не поддерживал попытки Планка объяснить спонтанное излучение эффектом случайного вмешательства, индуцированного фоновым полем. В то же время представление (11) квантовых средних соответствует квантовой экспериментальной науке, которая использует процедуру калибровки детекторов. Вычитание вклада случайного фона представляет собой теоретический аналог процедуры калибровки.

\section{5. РОЛЬ ФАЗЫ ВОЛНОВОЙ ФУНКЦИИ В КВАНТОВОЙ И ПРЕДКВАНТОВОЙ ТЕОРИЯХ}

В каноническом квантовом формализме вектор чистого состояния определен с точностью до фазового множителя. Два нормированных вектора $\Psi_{1}, \Psi_{2} \in H$ определяют одно и то же чистое квантовое состояние, тогда и только тогда, когда

$$
\Psi_{2}=e^{i \theta} \Psi_{1}
$$

Для системы, состоящей из одиночной частицы, то же самое происходит в рамках ПКСТП. Ковариационный оператор $D_{\Psi} \equiv \rho_{\Psi}=|\Psi\rangle\langle\Psi|$ предквантового случайного 
поля $\phi(\omega) \equiv \phi_{\Psi}(\omega)$ не зависит от фазового множителя, $D_{\Psi_{1}}=D_{\Psi_{2}}$, если $\Psi_{1}, \Psi_{2}$ связаны соотношением (12). Поэтому если мы ограничимся рассмотрением гауссовых предквантовых полей, то такие $\Psi_{1}$ и $\Psi_{2}$ определяют одно и то же поле. Однако для составных систем фазовый множитель играет совершенно другую роль. В КМ переход к составным системам ничего не меняет, в ПКСТП два вектора, связанные соотношением (12), определяют два различных случайных бисигнала (даже в гауссовом случае): они имеют ковариационные операторы с различными внедиагональными блоками. Заметим, что если два вектора из пространства $H_{1} \otimes H_{2}$ связаны соотношением (12), то соответствующие операторы связаны аналогичным соотношением $\widehat{\Psi}_{2}=e^{i \theta} \widehat{\Psi}_{1}$. Отсюда имеем

$$
D_{\Psi_{1}}=\left(\begin{array}{cc}
\widehat{\Psi}_{1} \widehat{\Psi}_{1}^{*}+\epsilon I & \widehat{\Psi}_{1} \\
\widehat{\Psi}_{1}^{*} & \widehat{\Psi}_{1}^{*} \widehat{\Psi}_{1}+\epsilon I
\end{array}\right), \quad D_{\Psi_{2}}=\left(\begin{array}{cc}
\widehat{\Psi}_{1} \widehat{\Psi}_{1}^{*}+\epsilon I & e^{i \theta} \widehat{\Psi}_{1} \\
e^{-i \theta} \widehat{\Psi}_{1}^{*} & \widehat{\Psi}_{1}^{*} \widehat{\Psi}_{1}+\epsilon I
\end{array}\right)
$$

Заметим, что диагональные блоки не зависят от фазы. Таким образом, в ПКСТП фаза нормированного вектора, представляющего чистое квантовое состояние (составной системы), не играет никакой роли для внутренних корреляций внутри каждой компоненты бисигнала $\phi=\left(\phi_{1}, \phi_{2}\right)$. Она важна только для корреляций между различными компонентами.

Пусть $\Psi \in H_{1} \otimes H_{2},\|\Psi\|^{2}=1$. Рассмотрим случайный бисигнал $\phi(\omega) \equiv \phi_{\Psi}(\omega)$ с ковариационным оператором $D \equiv D_{\Psi}$. Возьмем пару фаз $\gamma=\left(\gamma_{1}, \gamma_{2}\right)$, где $\gamma_{j} \in[0,2 \pi)$. Рассмотрим преобразование фаз в декартовом произведении гильбертовых пространств: $\Phi_{\gamma} \phi=\left(e^{i \gamma_{1}} \phi_{1}, e^{i \gamma_{2}} \phi_{2}\right)$. Теперь применим это преобразование к бисигналу $\phi(\omega)$, выходной сигнал задается как

$$
\phi_{\gamma}(\omega)=\Phi_{\gamma} \phi(\omega)
$$

ПРЕДЛОЖЕНИЕ 1. Ковариационный оператор выходного сигнала $\phi_{\gamma}$ имеет следуюшую структуру: его диагональные блоки такие же, как в случае сигнала ф, а внедиагональный блок имеет вид

$$
D_{12}^{\phi_{\gamma}}=e^{i\left(\gamma_{1}-\gamma_{2}\right)} D_{12}
$$

ДоказАтельство. Докажем, например, равенство (14): имеем

$$
\left\langle D_{12}^{\phi_{\gamma}} y_{2}, y_{1}\right\rangle=E\left\langle y_{2}, e^{i \gamma_{2}} \phi_{2}\right\rangle\left\langle e^{i \gamma_{1}} \phi_{1}, y_{1}\right\rangle=\left\langle D_{12} e^{-i \gamma_{2}} y_{2}, e^{-i \gamma_{1}} y_{1}\right\rangle
$$

Поскольку $D_{12}=\widehat{\Psi}$, выходной сигнал $\phi_{\gamma}$ соответствует вектору

$$
\Psi_{\gamma}=e^{i\left(\gamma_{1}-\gamma_{2}\right)} \Psi
$$

Таким образом, преобразование фаз бисигнала не меняет его распределение тогда и только тогда, когда относительная фаза $\gamma_{1}-\gamma_{2}=2 \pi k$.

Данный результат можно использовать для иллюстрации проблемы выбора фаз в ПКСТП. Пусть $\phi-$ бисигнал, соответствующий вектору $\Psi_{1}$. Тогда любой бисигнал $\phi_{\gamma}$ можно рассматривать как предквантовое представление того же квантового состояния. Таким образом, каждое чистое квантовое состояние в действительности представляет двупараметрическое семейство $\phi_{\gamma}$ предквантовых случайных полей 
(связанных преобразованиями фаз). Однако КМ об этом ничего не знает, поскольку на уровне квантовых наблюдаемых зависимость предквантовых случайных сигналов от фазы не проявляется.

В ПКСТП квантовые наблюдаемые представляются квадратичными формами предквантовых сигналов. Для пары самосопряженных операторов $\hat{A}_{j}: H_{j} \rightarrow H_{j}$ имеем $f_{A_{j}}\left(\phi_{\gamma, j}\right)=f_{A_{j}}\left(\phi_{j}\right), j=1,2$, где $\phi_{\gamma}=\left(\phi_{\gamma, 1}, \phi_{\gamma, 2}\right)$, поэтому

$$
E f_{A_{1}}\left(\phi_{\gamma, 1}\right) f_{A_{1}}\left(\phi_{\gamma, 2}\right)=E f_{A_{1}}\left(\phi_{1}\right) f_{A_{2}}\left(\phi_{2}\right) \text {. }
$$

Таким образом, на уровне корреляций квадратичных форм (и поэтому квантовых наблюдаемых) зависимость сигналов от фазы не проявляется.

\section{6. ПРЕОБРАЗОВАНИЕ КОВАРИАЦИОННОГО ОПЕРАТОРА ПРИ ПЕРЕСТАНОВКЕ КОМПОНЕНТ СИГНАЛА}

Рассмотрим оператор перестановки $\sigma\left(\phi_{1}, \phi_{2}\right)=\left(\phi_{2}, \phi_{1}\right)$ в декартовом произведении $H \times H$. Можно определить перестановочное преобразование предквантового случайного поля $\phi^{\sigma}(\omega)=\left(\phi_{2}(\omega), \phi_{1}(\omega)\right)$.

6.1. Бозонные предквантовые случайные сигналы. Естественно (по крайней мере для гауссовых сигналов) называть случайное поле симметричным относительно перестановок, или просто симметричным, если случайные поля $\phi$ и $\phi^{\sigma}$ имеют один и тот же ковариационный оператор. Однако определение ковариации включает операцию комплексного сопряжения. Чтобы соответствовать такому (математическому) определению ковариации, нужно дополнить перестановочное преобразование преобразованием сопряжения: $* \phi=\bar{\phi}$. Положим $\sigma_{*}=* \sigma$. Пусть $\phi(\omega)-$ случайное поле, рассмотрим его преобразование

$$
\phi^{\sigma_{*}}(\omega)=\sigma_{*} \phi(\omega)=\left(\bar{\phi}_{2}, \bar{\phi}_{1}\right) .
$$

Будем называть компоненты случайного бисигнала $\phi$ симметричными относительно перестановок или просто называть $\phi$ симметричным бисигналом, если $\phi$ и $\phi^{\sigma_{*}}$ имеют одинаковый ковариационный оператор (в гауссовом случае это означает совпадение распределений). Обозначим ковариационные операторы упомянутых выше бисигналов символами $D^{\phi}$ и $D^{\phi^{\sigma_{*}}}$. Таким образом, $\phi$ симметричен тогда и только тогда, когда $D^{\phi}=D^{\phi_{*}}$. Поскольку диагональные блоки этих операторов совпадают (см. подробности в разделе 7), данное условие сводится к совпадению внедиагональных блоков: $D_{12}^{\phi}=D_{12}^{\phi^{\sigma *}}$. Переформулируем это условие в терминах квантовых состояний. Пусть бисигнал $\phi$ соответствует нормированному вектору $\Psi$, тогда $\phi \equiv \phi_{\Psi}$.

ПРЕДЛОЖЕНИЕ 2. Бисигнал $\phi \equiv \phi_{\Psi}$, соответствующий чистому квантовому состоянию $\Psi$, является симметричным, если

$$
\left\langle\widehat{\Psi} \bar{y}_{1}, \bar{y}_{2}\right\rangle=\left\langle\widehat{\Psi} y_{2}, y_{1}\right\rangle \text {. }
$$

ДокАЗАТЕЛЬСтво. Найдем билинейную форму ковариаций для $\phi^{\sigma_{*}}$ :

$$
E\left\langle\phi_{1}^{\sigma_{*}}, y_{1}\right\rangle\left\langle\phi_{2}^{\sigma_{*}}, y_{2}\right\rangle=E\left\langle y_{2}, \bar{\phi}_{1}\right\rangle\left\langle\bar{\phi}_{2}, y_{1}\right\rangle=\left\langle D_{12} \bar{y}_{1}, \bar{y}_{2}\right\rangle=\left\langle\widehat{\Psi}_{\bar{y}_{1}}, \bar{y}_{2}\right\rangle,
$$

тем самым (17) доказано. 
Возьмем вещественный ортонормированный базис $\left\{e_{k}\right\}$ в $H, \bar{e}_{k}=e_{k}$. Тогда условие (17) эквивалентно следующему равенству для матричных элементов оператора $\widehat{\Psi}$ :

$$
\Psi_{i j}=\Psi_{j i}
$$

Если все матричные элементы вещественны, то этот оператор самосопряжен; в общем случае имеем более сложное ограничение для оператора $\widehat{\Psi}$ (см. раздел 7).

На самом деле мы не получили, что симметрия относительно перестановок эквивалентна самосопряженности чистого квантового состояния в операторном представлении, потому что комплексные структуры в тензорном произведении $H_{1} \otimes H_{2}$ и пространстве операторов $\mathcal{L}\left(H_{2}, H_{1}\right)$ не согласованны. Операторный вид равенства (17) будет представлен в разделе 7.

В вещественном базисе матричные элементы оператора $\widehat{\Psi}$ совпадают с координатами вектора $\Psi \in H_{1} \otimes H_{2}, \Psi=\sum_{i j} \Psi_{i j} e_{i} \otimes e_{j}$ (см. формулу (2)). Таким образом, в этом случае (19) можно интерпретировать как равенство координат вектора квантового состояния.

Пусть $H=L_{2}\left(\mathbb{R}^{3}\right)$. Здесь $\Psi=\Psi\left(x_{1}, x_{2}\right)$ - квадратично-интегрируемая функция на $\mathbb{R}^{6}$. Возьмем в $(19) y_{1}\left(u_{1}\right)=\delta\left(x_{1}-u_{1}\right)$ и $y_{2}\left(u_{2}\right)=\delta\left(x_{2}-u_{2}\right)$. Тогда получим $\Psi\left(x_{1}, x_{2}\right)=\Psi\left(x_{2}, x_{1}\right)$. Таким образом, предквантовый случайный сигнал (с одномерным проектором в качестве ковариационного оператора) симметричен, если он отвечает симметричной волновой функции. Соответствующая квантовая система “состоит из двух бозонов", поэтому можно назвать симметричные относительно перестановок предквантовые поля бозонными полями.

Для прояснения смысла равенства (17) можно использовать следующий пример. Рассмотрим чистое квантовое состояние вида

$$
\Psi=c_{1} \Psi_{1} \otimes \Psi_{2}+c_{2} \Psi_{2} \otimes \Psi_{1}
$$

где $\Psi_{1}, \Psi_{2} \in H, c_{1}, c_{2} \in \mathbb{C},\left|c_{1}\right|^{2}+\left|c_{2}\right|^{2}=1$. Тогда

$$
\begin{aligned}
\widehat{\Psi} y_{2} & =c_{1}\left\langle y_{2}, \bar{\Psi}_{2}\right\rangle \Psi_{1}+c_{2}\left\langle y_{2}, \bar{\Psi}_{1}\right\rangle \Psi_{2}, \\
\left\langle\widehat{\Psi} y_{2}, y_{1}\right\rangle & =c_{1}\left\langle y_{2}, \bar{\Psi}_{2}\right\rangle\left\langle\Psi_{1}, y_{1}\right\rangle+c_{2}\left\langle y_{2}, \bar{\Psi}_{1}\right\rangle\left\langle\Psi_{2}, y_{1}\right\rangle, \\
\left\langle\widehat{\Psi} \bar{y}_{1}, \bar{y}_{2}\right\rangle & =c_{1}\left\langle\bar{y}_{1}, \bar{\Psi}_{2}\right\rangle\left\langle\Psi_{1}, \bar{y}_{2}\right\rangle+c_{2}\left\langle\bar{y}_{1}, \bar{\Psi}_{1}\right\rangle\left\langle\Psi_{2}, \bar{y}_{2}\right\rangle= \\
& =c_{1}\left\langle\Psi_{2}, y_{1}\right\rangle\left\langle y_{2}, \bar{\Psi}_{1}\right\rangle+c_{2}\left\langle\Psi_{1}, y_{1}\right\rangle\left\langle y_{2}, \bar{\Psi}_{2}\right\rangle .
\end{aligned}
$$

Сравнивая эти выражения, получаем равенство $c_{1}=c_{2}=1 / \sqrt{2}$, т. е.

$$
\widehat{\Psi}=\frac{1}{\sqrt{2}}\left(\Psi_{1} \otimes \Psi_{2}+\Psi_{2} \otimes \Psi_{1}\right) .
$$

6.2. Фермионные предквантовые случайные сигналы. Будем называть компоненты случайного бисигнала ф антисимметричными относительно перестановок или просто называть $\phi$ антисимметричным бисигналом, если ковариационные операторы $\phi$ и $\phi^{\sigma_{*}}$ имеют одинаковые диагональные блоки, а внедиагональные блоки имеют различные знаки: $D_{12}^{\phi^{\sigma *}}=-D_{12}^{\phi}$. 
ПРЕДЛОЖЕНИЕ 3. Бисигнал $\phi \equiv \phi_{\Psi}$, соответствующий чистому квантовому состоянию $\Psi$, является антисимметричным, если

$$
\left\langle\widehat{\Psi} \bar{y}_{1}, \bar{y}_{2}\right\rangle=-\left\langle\widehat{\Psi} y_{2}, y_{1}\right\rangle \text {. }
$$

ДокАЗАТЕЛЬСТво этого предложения аналогично доказательству предложения 2.

Для вещественного базиса в $H$ это условие эквивалентно следующему соотношению для матричных элементов оператора $\widehat{\Psi}$ (координат вектора состояния $\Psi$ ): $\Psi_{i j}=-\Psi_{j i}$. Если все матричные элементы вещественны, то это эквивалентно кососимметричности оператора $\widehat{\Psi}$.

Теперь возьмем $H=L_{2}\left(\mathbb{R}^{3}\right)$. Аналогично тому, как мы делали в п. 6.1, получим, что волновая функция $\Psi\left(x_{1}, x_{2}\right)$ антисимметричного бисигнала $\phi$ является антисимметричной: $\Psi\left(x_{1}, x_{2}\right)=-\Psi\left(x_{2}, x_{1}\right)$. Соответствующая квантовая система "состоит из двух фермионов", поэтому можно называть антисимметричные относительно перестановок предквантовые поля фермионными полями.

Рассмотрим чистое квантовое состояние вида (20). Соответствующий случайный бисигнал $\phi$ является антисимметричным тогда и только тогда, когда $c_{1}=-c_{2}$, т. е.

$$
\widehat{\Psi}=\frac{1}{\sqrt{2}}\left(\Psi_{1} \otimes \Psi_{2}-\Psi_{2} \otimes \Psi_{1}\right) .
$$

Теперь изменим преобразование $\sigma_{*}$, положив

$$
\phi^{\sigma_{*-}} \equiv \sigma_{*-} \phi=\left(-\bar{\phi}_{2}, \bar{\phi}_{1}\right) .
$$

Бисигнал является антисимметричным тогда и только тогда, когда сигналы $\phi$ и $\phi^{\sigma_{*-}}$ имеют одинаковые ковариационные операторы, $D^{\phi}=D^{\phi^{\sigma_{*-}}}$, что эквивалентно совпадению внедиагональных блоков, $D_{12}^{\phi}=D_{12}^{\phi^{\sigma_{*-}}}$.

Таким образом, предквантовое случайное поле является фермионным, если его ковариационный оператор не изменяется после перестановок компонент и изменения фазы на $\Delta \gamma=\pi$. Учитывая результат раздела 5 , мы видим, что на самом деле важно только изменение относительной фазы компонент сигнала. Вместо преобразования (22) можно рассмотреть преобразование (здесь $\left.\gamma=\left(\gamma_{1}, \gamma_{2}\right)\right)$

$$
\phi^{\sigma_{* \gamma}} \equiv \sigma_{*} \Phi_{\gamma} \phi=\left(e^{-i \gamma_{2}} \bar{\phi}_{2}, e^{-i \gamma_{1}} \bar{\phi}_{1}\right),
$$

где $\Delta \gamma=\gamma_{1}-\gamma_{2}=\pi$.

ПКСТП представляет собой формальный классический полевой формализм для квантовых явлений. Он не может объяснить, почему один тип сигналов проявляет симметрию своих компонент, а другой - антисимметрию. Заметим, что КМ также не может объяснить, почему одни частицы являются бозонами, а другие - фермионами. В КМ статистики Бозе и Ферми часто связывают с неразличимостью квантовых систем. Рассмотрим бозонный бисигнал; из симметрии его компонент относительно перестановок не следует неразличимость. В принципе мы можем надеяться, что улучшение нашей техники позволит проследить за компонентами сигнала и, таким образом, выделить их (аналогично тому, как это делается для классических частиц). Из симметрии относительно перестановок следует только то, что ковариационный 
оператор инвариантен относительно обмена компонентами сигнала (или, в гауссовом случае, что распределение вероятностей является симметричным). Из антисимметрии относительно перестановок следует, что ковариационный оператор инвариантен относительно обмена компонентами сигнала в комбинации со сдвигом на $\pi$ относительной фазы компонент.

Можно также рассмотреть предквантовые случайные сигналы анионного типа. Рассмотрим преобразование сигнала (23), где $\Delta \gamma=\theta$, угол $\theta \in[0,2 \pi)$ фиксирован. Будем называть $\phi$ анионным бисигналом с $\theta$-симметрией, если бисигналы $\phi$ и $\phi^{\sigma_{* \gamma_{1}} \gamma_{2}}$ имеют одинаковые ковариационные операторы (в гауссовом случае - одинаковое распределение) или (эквивалентно) бисигналы $\phi$ и $\phi^{\sigma_{*}}$ имеют одинаковые диагональные блоки, а внедиагональные блоки связаны соотношением $D_{12}^{\phi}=e^{i \theta} D_{12}^{\phi^{\sigma_{*}}}$. Из формализма ПКСТП не следует, что анионные поля для $\theta \neq \pi k$ не существуют.

\section{7. ОПЕРАТОРНОЕ ПРЕДСТАВЛЕНИЕ УСЛОВИЙ СИММЕТРИИ И АНТИСИММЕТРИИ ОТНОСИТЕЛЬНО ПЕРЕСТАНОВОК}

Используя операцию комплексного сопряжения (3) в пространстве операторов, равенство (18) можно записать как $D_{12}^{\phi^{\sigma_{*}}}=\widehat{\bar{\Psi}}^{*}$, а равенство $(17)-$ как

$$
\widehat{\Psi}=\widehat{\bar{\Psi}}^{*}
$$

или

$$
\widehat{\bar{\Psi}}=\widehat{\Psi}^{*} .
$$

Это операторное представление условия симметрии относительно перестановок.

Теперь покажем (как мы обещали в п. 6.1), что для бозонного бисигнала диагональные блоки его ковариационного оператора не изменяются при перестановочном преобразовании (16). Имеем

$$
\left\langle D_{11}^{\phi_{\sigma *}} y_{1}, y_{1}\right\rangle=E\left\langle y_{1}, \bar{\phi}_{2}\right\rangle\left\langle\bar{\phi}_{2}, y_{1}\right\rangle=\left\langle D_{22} \bar{y}_{1}, \bar{y}_{1}\right\rangle=\left\langle\bar{D}_{22}^{\phi} y_{1}, y_{1}\right\rangle
$$

Таким образом, равенство $D_{11}^{\phi^{\sigma_{*}}}=D_{11}^{\phi}$ можно записать как $D_{11}^{\phi}=\bar{D}_{22}^{\phi}$. Имеем $D_{11}^{\phi}=\widehat{\Psi} \widehat{\Psi}^{*}$ и $\bar{D}_{22}^{\phi}=\widehat{\bar{\Psi}}^{*} \widehat{\bar{\Psi}}$. С учетом (25) получаем $\bar{D}_{22}^{\phi}=\widehat{\Psi} \widehat{\Psi}^{*}$.

Аналогично, в фермионном случае условие (21) можно записать как $\widehat{\Psi}=-\widehat{\bar{\Psi}}^{*}$. Это операторное представление условия антисимметрии относительно перестановок. Ясно, что при таком условии диагональные блоки ковариационного оператора сохраняются (как и в случае симметрии относительно перестановок).

Мы снова утверждаем (см. раздел 5), что любое чистое квантовое состояние определяет двухпараметрическое семейство предквантовых (гауссовых) случайных полей $\phi_{\gamma}=\Phi_{\gamma} \phi, \gamma=\left(\gamma_{1}, \gamma_{2}\right)$ (см. формулу (13)), где $\phi \equiv \phi_{\Psi}-$ случайное поле с ковариационным оператором $D_{\Psi}$. Покажем, что если один из представителей этого семейства случайных полей симметричен относительно перестановок (бозонное поле), то все случайные поля $\phi_{\gamma}$ также являются бозонными. Предположим, что, например, $\phi \equiv \phi_{\Psi}$ является симметричным, т. е. выполнено соотношение (24). Согласно (15) случайное поле $\phi_{\gamma}$ соответствует вектору $\Psi_{\gamma}=e^{i \theta} \Psi$, где $\theta=\gamma_{1}-\gamma_{2}$. Заметим, что для любого оператора $\widehat{C}$ и его фазового сдвига $\widehat{C}_{\theta}=e^{i \theta} \widehat{C}$ имеет место равенство $\widehat{\bar{C}}_{\theta}=e^{-i \theta} \widehat{\bar{C}}$. Нам также известно, что $\widehat{C}_{\theta}^{*}=e^{-i \theta} \widehat{C}^{*}$. Поэтому если 
выполнено условие $(24)$, то $\widehat{\bar{\Psi}}_{\gamma}^{*}=\widehat{\Psi}_{\gamma}$. Таким образом, случайное поле $\phi_{\gamma}$ является симметричным.

Антисимметричные случайные поля обладают таким же свойством, и каждое бозонное (фермионное) квантовое состояние определяет семейство бозонных (фермионных) предквантовых случайных полей. Их невозможно различить на уровне квантовомеханической модели.

\section{8. ПРЕДСТАВЛЕНИЕ КВАНТОВЫХ КАНАЛОВ В ВИДЕ КЛАССИЧЕСКИХ КАНАЛОВ}

Как показано в работе [36], классического гауссовы каналы можно представить как квантовые каналы (этот факт использовался в классической теории сигналов). В работе [46] показано, что некоторые важные квантовые каналы можно представлять как классические каналы (при этом неясно, каждый ли квантовый канал можно представить как классический). В настоящей работе нас интересует классическое представление квантовых каналов, соответствующих унитарным преобразованиям.

Уравнение Шредингера с точки зрения классического случайного поля. Прежде чем перейти к динамике ПКСТП, рассмотрим уравнение Шредингера в стандартном формализме KM:

$$
i h \frac{\partial \Psi}{\partial t}(t)=\widehat{\mathcal{H}} \Psi(t), \quad \Psi\left(t_{0}\right)=\Psi_{0},
$$

где $\widehat{\mathcal{H}}$ - гамильтониан.

Напомним, что зависящее от времени случайное поле $\phi(t, x, \omega)$ называется стохастическим процессом (с пространством состояний $H$ ). Динамика предквантового случайного поля описывается простейшим стохастическим процессом, который определяется детерминированной динамикой со случайными начальными условиями. В ПКСТП уравнение Шредингера со случайным начальным условием описывается динамикой предквантового случайного поля, т. е. предквантовый стохастический процесс можно получить из "представления в виде декартова произведения" для уравнения Шредингера:

$$
i h \frac{\partial \phi}{\partial t}(t, \omega)=\widehat{\mathcal{H}}_{\mathrm{PCSFT}} \phi(t, \omega), \quad \phi\left(t_{0}, \omega\right)=\phi_{0}(\omega) .
$$

Здесь $\widehat{\mathcal{H}}_{\text {PCSFT }}: H_{1} \times H_{2} \rightarrow H_{1} \times H_{2}$ - генератор динамики ПКСТП, соответствующий квантовому генератору $\widehat{\mathcal{H}}: H_{1} \otimes H_{2} \rightarrow H_{1} \otimes H_{2}$. Начальное случайное поле $\phi_{0}(\omega)=\left(\phi_{01}(\omega), \phi_{02}(\omega)\right)$ определяется чистым квантовым состоянием $\Psi_{0}$. Это поле с нулевым средним значением и ковариационным оператором $D_{\Psi_{0}}$, заданным по формуле (8).

Для квантового гамильтониана без взаимодействия

$$
\widehat{\mathcal{H}}=\widehat{\mathcal{H}}_{1} \otimes I+I \otimes \widehat{\mathcal{H}}_{2}
$$

нетрудно найти его образ в ПКСТП: $\widehat{\mathcal{H}}_{\text {РСSFт }}=\widehat{\mathcal{H}}_{1} \times \widehat{\mathcal{H}}_{2}$, т. е. динамику $(26)$ можно записать в виде системы уравнений для компонент случайного поля

$$
\begin{aligned}
i h \frac{\partial \phi_{1}}{\partial t}(t, \omega) & =\widehat{\mathcal{H}}_{1} \phi_{1}(t, \omega), & i h \frac{\partial \phi_{2}}{\partial t}(t, \omega) & =\widehat{\mathcal{H}}_{2} \phi_{2}(t, \omega), \\
\phi_{1}\left(t_{0}, \omega\right) & =\phi_{01}(\omega), & \phi_{2}\left(t_{0}, \omega\right) & =\phi_{02}(\omega) .
\end{aligned}
$$


Положим $U_{j t}=e^{-i t \widehat{\mathcal{H}}_{j} / h}, j=1,2$, и

$$
V_{t}=U_{1 t} \times U_{2 t}
$$

Тогда $\phi_{t}=V_{t} \phi_{0}$. Нетрудно видеть, что внедиагональный блок случайного поля $\phi_{t}(\omega)=V_{t} \phi_{0}(\omega)$ дается оператором $\widehat{\Psi_{t}}$, где $\Psi_{t}=U_{t} \Psi_{0}$ и $U_{t}=U_{1 t} \otimes U_{2 t}$. Таким образом, уравнение (26) для классического случайного процесса согласованно с уравнением Шредингера в КМ.

Заметим, что несмотря на представление (28) мы не можем разделить динамику (27) (как динамику случайных полей) на две независимые динамики для компонент случайного поля. Вообще говоря, начальные поля $\phi_{01}(\omega)$ и $\phi_{02}(\omega)$ не являются независимыми.

Любой квантовый канал, задаваемый унитарным оператором $U=U_{1} \otimes U_{2}$, можно представить как унитарное преобразование классического случайного сигнала:

$$
\phi_{\text {out }}(\omega)=\left(U_{1} \phi_{\text {in }, 1}(\omega), U_{2} \phi_{\text {in }, 2}(\omega)\right) .
$$

Для входящего сигнала, соответствующего чистому квантовому состоянию $\Psi_{\text {in }}$, внедиагональный блок канала $\phi_{\text {out }}$ задается выражением

$$
\Psi_{\text {out }}=U \Psi_{\text {in }}
$$

\section{9. ГРУППИРОВАНИЕ И АНТИГРУППИРОВАНИЕ В КВАНТОВОЙ ТЕОРИИ}

Рассмотрим следующий эксперимент. Две тождественные квантовые частицы падают на светоделитель в отношении $1: 1$, и мы измеряем выходной сигнал с помощью двух счетчиков частиц. Для анализа этой задачи пометим падающие частицы как частицу 1 и частицу 2, основываясь на "щелчках" детекторов. Одним из возможных состояний на входе является состояние $|1 \mathrm{R}\rangle|2 \mathrm{~L}\rangle$ : частица 1 находится в правом канале входа, а частица 2 - в левом. Поскольку тождественные квантовые частицы являются "неразличимыми", также возможно состояние $|1 \mathrm{~L}\rangle|2 \mathrm{R}\rangle$ на входе: частица 1 на левом входе, а частица 2 - на правом.

Таким образом, наиболее общее состояние на входе строится как линейная суперпозиция двух состояний: $\Psi=c_{1}|1 \mathrm{R}\rangle|2 \mathrm{~L}\rangle+c_{2}|1 \mathrm{~L}\rangle|2 \mathrm{R}\rangle$, где $\left|c_{1}\right|^{2}+\left|c_{2}\right|^{2}=1$, если два состояния ортогональны. Физическое состояние, представляющее реальную систему, состоящую из тождественных квантовых частиц, является или симметричным, или антисимметричным относительно перестановки любых двух частиц (экспериментальный факт): для бозонов

$$
\Psi=\frac{1}{\sqrt{2}}(|1 \mathrm{R}\rangle|2 \mathrm{~L}\rangle+|1 \mathrm{~L}\rangle|2 \mathrm{R}\rangle)
$$

а для фермионов

$$
\Psi=\frac{1}{\sqrt{2}}(|1 \mathrm{R}\rangle|2 \mathrm{~L}\rangle-|1 \mathrm{~L}\rangle|2 \mathrm{R}\rangle) .
$$

Формализм КМ предсказывает, что взаимодействие двухбозонной системы со светоделителем приведет к эффекту группирования; в фермионном случае получится 
эффект антигруппирования. Наша цель состоит в том, чтобы предложить модель этих эффектов в рамках классической теории сигналов. Мы покажем, что предквантовые бисигналы с компонентами, симметричными относительно перестановок, дают группирование, а с антисимметричными компонентами - антигруппирование.

\section{0. ВЗАИМОДЕЙСТВИЕ КЛАССИЧЕСКОГО СИГНАЛА СО СВЕТОДЕЛИТЕЛЕМ}

Рассмотрим классический случайный сигнал $\chi$, взаимодействующий со светоделителем; одна часть сигнала $\chi$ проходит через левый вход светоделителя и обозначается как $\chi_{\mathrm{L}}$, а другая - через правый и обозначается как $\chi_{\mathrm{R}}$. Таким образом, $\chi=\left(\chi_{\mathrm{L}}, \chi_{\mathrm{R}}\right)$. Из классической электродинамики известно, что для входного сигнала $\chi_{\mathrm{in}}=\left(\chi_{\mathrm{in}, \mathrm{L}}, \chi_{\mathrm{in}, \mathrm{R}}\right)$ выходной сигнал $\chi_{\text {out }}=\left(\chi_{\text {out }, \mathrm{L}}, \chi_{\mathrm{out}, \mathrm{R}}\right)$, где индексы L и $\mathrm{R}$ теперь используются для выходов светоделителя, можно получить в виде $\chi_{\text {out }}(\omega)=U \chi_{\text {in }}(\omega)$, где для светоделителя в отношении 1:1 преобразование $U$ представляет собой вращение на угол $\pi / 4$ (классический закон Малюса):

$$
U=\frac{1}{\sqrt{2}}\left(\begin{array}{cc}
1 & -1 \\
1 & 1
\end{array}\right) .
$$

Теперь возьмем бисигнал $\phi_{\text {in }}=\left(\phi_{\text {in }, 1}, \phi_{\text {in }, 2}\right)$, у которого каждая из его компонент $\phi_{\mathrm{in}, i}=\left(\phi_{\mathrm{in}, i, \mathrm{~L}}, \phi_{\mathrm{in}, i, \mathrm{R}}\right), i=1,2$, преобразуется по закону Малюса (33). Выходной бисигнал имеет вид $\phi_{\text {out }}(\omega)=(U \times U) \phi_{\text {in }}(\omega)$ (см. формулу $\left.(29)\right)$. Мы рассматриваем внедиагональный блок ковариационного оператора для $\phi_{\text {out }}$. В соответствии с (30) он равен $\Psi_{\text {out }}=(U \otimes U) \Psi_{\text {in }}$, поэтому для бозоннного входного бисигнала, т. е. для

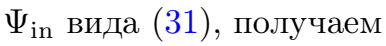

$$
\Psi_{\text {out }}=\frac{1}{\sqrt{2}}(|1 \mathrm{R}\rangle|2 \mathrm{R}\rangle-|1 \mathrm{~L}\rangle|2 \mathrm{~L}\rangle) .
$$

Для фермионного входного бисигнала, т. е. для $\Psi_{\text {in }}$ вида $(32)$, получаем

$$
\Psi_{\text {out }}=\frac{1}{\sqrt{2}}(|1 \mathrm{R}\rangle|2 \mathrm{~L}\rangle-|1 \mathrm{~L}\rangle|2 \mathrm{R}\rangle) .
$$

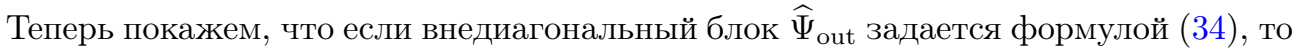
мы имеем группирование классических сигналов, а из формулы (35) следует антигруппирование. Рассмотрим последний случай, т. е. фермионный бисигнал. Положим $\phi \equiv \phi_{\text {out }}$. Интенсивности компонент сигнала в точках $x=\mathrm{R}, \mathrm{L}$ даются выражениями $I_{i x}=\left|\phi_{i x}\right|^{2}, i=1,2$, а их ковариации равны

$$
g_{x y}=\left\langle\left(I_{1 x}-\left\langle I_{1 x}\right\rangle\right)\left(I_{2 y}-\left\langle I_{2 y}\right\rangle\right)\right\rangle, \quad x=\mathrm{R}, \mathrm{L} .
$$

Введем операторы (проекторы) $\hat{A}_{x}=|x\rangle\langle x|, x=\mathrm{R}, \mathrm{L}$. Тогда $\left|\phi_{i x}\right|^{2}=\left\langle\hat{A}_{x} \phi_{i}, \phi_{i}\right\rangle$ (это интенсивность $i$-й компоненты бисигнала, $i=1,2$, на выходе $x=\mathrm{R}, \mathrm{L}$ ).

Таким образом, согласно основному равенству (4) имеем

$$
g_{\mathrm{RR}}=\left\langle\left(\hat{A}_{\mathrm{R}} \otimes \hat{A}_{\mathrm{R}}\right) \Psi_{\text {out }}, \Psi_{\text {out }}\right\rangle, \quad g_{\mathrm{RL}}=\left\langle\left(\hat{A}_{\mathrm{R}} \otimes \hat{A}_{\mathrm{L}}\right) \Psi_{\text {out }}, \Psi_{\text {out }}\right\rangle .
$$


Для фермионного $\Psi_{\text {out }}(35)$ получаем $g_{\mathrm{RR}}=0<g_{\mathrm{RL}}=1 / 2$, и мы имеем явление антигруппирования. В бозонном случае получаем $g_{\mathrm{RL}}=0<g_{\mathrm{RR}}=1 / 2$, и мы имеем явление группирования.

На самом деле здесь нет ничего удивительного. Поскольку мы связываем корреляции классического поля с квантовыми корреляциями (см. соотношение (4)), можно ожидать, что все квантовые эффекты будут воспроизведены.

\section{1. ВЕКТОРНОЗНАЧНЫЕ ПРЕДКВАНТОВЫЕ ПОЛЯ, ВНУТРЕННИЕ СТЕПЕНИ СВОБОДЫ}

Пусть $H_{\text {space }}=L_{2}\left(\mathbb{R}^{3}\right)$ - пространство комплекснозначных функций, квадратично-суммируемых по мере Лебега $d x=d x_{1} d x_{2} d x_{3}$; пусть $H_{\text {internal }}=\mathbb{C}^{n}$ - пространство внутренних степеней свободы. Теперь рассмотрим векторные поля $\phi(x)=$ $\left(\phi^{1}(x), \ldots, \phi^{n}(x)\right)$, где каждое поле $\phi^{j}(x)$ принадлежит $H_{\text {space }}$ Таким образом, $\phi$ принадлежит $L_{2}\left(\mathbb{R}^{3} ; \mathbb{C}^{n}\right)$, т. е. пространству $\mathbb{C}^{n}$-значных квадратично-суммируемых функций (с квадратом нормы $\left.\|\phi\|^{2}=\int \sum_{j}\left|\phi^{j}(x)\right|^{2} d x\right)$. Заметим, что

$$
H_{1}=L_{2}\left(\mathbb{R}^{3} ; \mathbb{C}^{n}\right)=H_{\text {space }} \otimes H_{\text {internal }}
$$

Таким образом, любой нормированный вектор $\Psi \in H_{\text {space }} \otimes H_{\text {internal }}$ определяет предквантовое случайное векторное поле с ковариационным оператором $D=|\Psi\rangle\langle\Psi|$.

Рассмотрим случайные бисигналы с компонентами $\phi_{i}(x)=\left(\phi_{i}^{1}(x), \ldots, \phi_{i}^{n}(x)\right), i=$ 1,2 , соответствующие чистому квантовому состоянию $\Psi \in L_{2}\left(\mathbb{R}^{3} ; \mathbb{C}^{n}\right) \otimes L_{2}\left(\mathbb{R}^{3} ; \mathbb{C}^{n}\right)$. $\mathrm{C}$ математической точки зрения это состояние можно рассматривать как вектор, лежащий в тензорном произведении

$$
H_{12}=H_{\text {space }} \otimes H_{\text {internal }} \otimes H_{\text {space }} \otimes H_{\text {internal }} .
$$

Более того, с математической точки зрения может оказаться удобным разделить пространственные и внутренние координаты и представить $H_{12}$ в виде

$$
H_{12}=\left(H_{\text {space }} \otimes H_{\text {space }}\right) \otimes\left(H_{\text {internal }} \otimes H_{\text {internal }}\right) \text {. }
$$

Заметим, что это всего лишь математическое представление.

Предложенную конструкцию можно обобщить на пространство $L_{2}(X, \nu)$, где $X-$ множество, а $\nu$ - мера на этом множестве; здесь $\|\phi\|^{2}=\int_{X}|\phi(x)|^{2} d \nu(x)$. На самом деле мы рассматриваем простой случай, в котором $X=\left\{x_{1}, \ldots, x_{m}\right\}-$ конечное множество, а $\nu$ - равномерная мера на $X$, т. е. $\nu(x)=1$ для любого $x$. При этом вместо интеграла мы имеем сумму, $\|\phi\|^{2}=\sum_{k}\left|\phi\left(x_{k}\right)\right|^{2}$, пространство является дискретным и $H_{\text {space }}=\mathbb{C}^{m}$. С учетом внутреннних степеней свободы получаем тензоры $\phi=\left(\phi^{j i}\right), j=1, \ldots, m, i=1, \ldots, n$, где первый индекс описывает пространственные степени свободы, а второй - внутренние. Отсюда пространство (37) имеет вид $H_{1}=\mathbb{C}^{m} \otimes \mathbb{C}^{n}$. Для бисигнала имеем

$$
H_{12}=\left(\mathbb{C}^{m} \otimes \mathbb{C}^{n}\right) \otimes\left(\mathbb{C}^{m} \otimes \mathbb{C}^{n}\right) .
$$

При чисто математическом рассмотрении можно работать с пространством

$$
H_{12}=\left(\mathbb{C}^{m} \otimes \mathbb{C}^{m}\right) \otimes\left(\mathbb{C}^{n} \otimes \mathbb{C}^{n}\right) .
$$




\section{2. ГРУППИРОВАНИЕ И АНТИГРУППИРОВАНИЕ: ПРЕДКВАНТОВЫЕ ПОЛЯ СО СПИНОМ}

В нашем формализме квантовая система со спином $1 / 2$ описывается следующим образом.

Возьмем $X=\{\mathrm{R}, \mathrm{L}\}$, т. е. в данном случае $m=2$. Пусть $n=2$ (две внутренние степени свободы), $H_{\text {space }}=\mathbb{C}^{2}, H_{\text {internal }}=\mathbb{C}^{2}$. Получаем $\mathbb{C}^{2}$-значные "поля" $\phi=\left(\phi^{+}(\mathrm{R}), \phi^{-}(\mathrm{R}), \phi^{+}(\mathrm{L}), \phi^{-}(\mathrm{L})\right)$. Такой случайный вектор описывает квантовую систему со спином $1 / 2$. Возьмем, например, вектор $\psi=|\mathrm{R}\rangle|+\rangle$. Он определяет случайное предквантовое поле со спином $1 / 2$. Теперь рассмотрим два таких поля бисигнал с компонентами

$$
\phi_{i}=\left(\phi_{i}^{+}(\mathrm{R}), \phi_{i}^{-}(\mathrm{R}), \phi_{i}^{+}(\mathrm{L}), \phi_{i}^{-}(\mathrm{L})\right), \quad i=1,2 .
$$

Рассмотрим вектор

$$
\Psi=\frac{1}{2}(|1 \mathrm{R}\rangle|2 \mathrm{~L}\rangle-|1 \mathrm{~L}\rangle|2 \mathrm{R}\rangle) \otimes\left(|+\rangle_{1}|-\rangle_{2}-|-\rangle_{1}|+\rangle_{2}\right)
$$

Это чисто математическое представление (см. представление (38)). Чтобы следовать ПКСТП, перепишем вектор как

$$
\begin{aligned}
\Psi= & \frac{1}{2}\left(|1 \mathrm{R}\rangle|+\rangle_{1} \otimes|2 \mathrm{~L}\rangle|-\rangle_{2}-|1 \mathrm{R}\rangle|-\rangle_{1} \otimes|2 \mathrm{~L}\rangle|+\rangle_{2}-\right. \\
& \left.-|1 \mathrm{~L}\rangle|+\rangle_{1} \otimes|2 \mathrm{R}\rangle|-\rangle_{2}+|1 \mathrm{~L}\rangle|-\rangle_{1} \otimes|2 \mathrm{R}\rangle|+\rangle_{2}\right) .
\end{aligned}
$$

Этот вектор $\Psi \in\left(H_{\text {space }} \otimes \mathbb{C}^{2}\right) \otimes\left(H_{\text {space }} \otimes \mathbb{C}^{2}\right)$ определяет внедиагональный блок матрицы ковариаций гауссова бисигнала с компонентами, принимающими значения в $H_{\text {space }} \otimes \mathbb{C}^{2}-$ пространстве полей, действующих из $H_{\text {space }}$ в $\mathbb{C}^{2}$.

Интенсивности компонент сигнала в точках $x=\mathrm{R}, \mathrm{L}$ имеют вид

$$
I_{i x}=\left|\phi_{i}^{+}(x)\right|^{2}+\left|\phi_{i}^{-}(x)\right|^{2}, \quad i=1,2,
$$

а их ковариации равны $g_{x y}=\left\langle\left(I_{1 x}-\left\langle I_{1 x}\right\rangle\right)\left(I_{2 y}-\left\langle I_{2 y}\right\rangle\right)\right\rangle$, где $x, y=\mathrm{R}, \mathrm{L}$.

Введем операторы (проекторы) $\hat{A}_{1 x}=|x\rangle\langle x| \otimes I$ и $\hat{A}_{2 x}=I \otimes|x\rangle\langle x|$. Соответствующие квадратичные формы (физические переменные ПКСТП) даются выражением

$$
f_{A_{i x}}\left(\phi_{i}\right)=\left|\phi_{i}^{+}(x)\right|^{2}+\left|\phi_{i}^{-}(x)\right|^{2}=I_{i x}, \quad i=1,2, \quad x=\mathrm{R}, \mathrm{L} .
$$

Как и в случае бесспиновых полей (36), определим ковариации интенсивностей. Согласно основному равенству, выражающему связь предквантовых и квантовых корреляций, получаем

$$
\begin{aligned}
g_{\mathrm{RR}} & =\left\langle\left(f_{A_{1 \mathrm{R}}}-\left\langle f_{A_{1 \mathrm{R}}}\right\rangle\right)\left(f_{A_{2 \mathrm{R}}}-\left\langle f_{A_{2 \mathrm{R}}}\right\rangle\right)\right\rangle=\left\langle\hat{A}_{1 \mathrm{R}} \otimes \hat{A}_{2 \mathrm{R}} \Psi, \Psi\right\rangle= \\
& =\left\langle\left(\hat{A}_{\mathrm{R}} \otimes \hat{A}_{\mathrm{R}}\right) \Psi_{\text {space }}, \Psi_{\text {space }}\right\rangle\left\|\Psi_{\text {spin }}\right\|^{2}=0,
\end{aligned}
$$

где

$$
\Psi_{\text {space }}=\frac{1}{\sqrt{2}}(|1 \mathrm{R}\rangle|2 \mathrm{~L}\rangle-|1 \mathrm{~L}\rangle|2 \mathrm{R}\rangle), \quad \Psi_{\text {spin }}=\frac{1}{\sqrt{2}}\left(|+\rangle_{1}|-\rangle_{2}-|-\rangle_{1}|+\rangle_{2}\right)
$$


(мы снова утверждаем, что последние векторы представляют собой чисто математические выражения; физически пространственные компоненты связаны со спиновыми).

Таким образом, в полном согласии с формальными предсказаниями КМ классические бозонные (симметричные относительно перестановок) бисигналы со спином $1 / 2$ демонстрируют свойство “фермионного столкновения": $g_{\mathrm{RR}}=0$. Если мы рассмотрим фермионный бисигнал со спином $1 / 2$ такой, что корреляции внутренних компонент определяются оператором $\widehat{\Psi}$, соответствующим вектору, который можно формально записать как

$$
\Psi=\frac{1}{2}(|1 \mathrm{R}\rangle|2 \mathrm{~L}\rangle+|1 \mathrm{~L}\rangle|2 \mathrm{R}\rangle) \otimes\left(|+\rangle_{1}|-\rangle_{2}-|-\rangle_{1}|+\rangle_{2}\right)
$$

то, снова в полном согласии с формальными предсказаниями КМ, такой бисигнал демонстрирует свойство "бозонного столкновения": $g_{\mathrm{RL}}=0$.

Вывод. Группирование и антигруппирование для квантовых систем можно представить в рамках классических сигналов.

\section{Список литературы}

[1] A. Plotnitsky, J. Modern Optics, 54:16-17 (2007), 2393-2402.

[2] A. Plotnitsky, Epistemology and Probability: Bohr, Heisenberg, Schrödinger, and the Nature of Quantum-Theoretical Thinking, Fundamental Theories of Physics, 161, Springer, Berlin, New York, NY, 2009.

[3] W. H. Louisell, Quantum Statistical Properties of Radiation, Reprint of the 1973 edition, Wiley \& Sons, New York, 1990.

[4] L. Mandel, E. Wolf, Optical Coherence and Quantum Optics, Cambridge Univ. Press, Cambridge, 1995.

[5] M. O. Scully, M. S. Zubairy, Quantum Optics, Cambridge Univ. Press, Cambridge, 1997.

[6] L. de la Pea, A. Cetto, The Quantum Dice: an Introduction to Stochastic Electrodynamics, Fundamental Theories of Physics, 75, Kluwer, Dordrecht, 1996.

[7] A. Casado, T. Marshall, E. Santos, J. Opt. Soc. Am. B, 14:3 (1997), 494-502.

[8] G. Brida, M. Genovese, M. Gramegna, C. Novero, E. Predazzi, Phys. Lett. A, 299:2-3 (2002), 121-124, arXiv: quant-ph/0203048.

[9] T. H. Boyer, "A brief survey of stochastic electrodynamics", Foundations of Radiation Theory and Quantum Electrodynamics, ed. A. Barut, Plenum, New York, London, 1980, 49-63.

[10] D. C. Cole, A. Rueda, K. Danley, Phys. Rev. A, 63:5 (2001), 054101, 2 pp.

[11] Th. M. Nieuwenhuizen, "Classical phase space density for the relativistic hydrogen atom", Quantum Theory: Reconsideration of Foundations, Proceedings of the 3rd International Conference (Växjö, Sweden, June 6-11, 2005), AIP Conference Proceedings, 810, eds. G. Adenier, A. Yu. Khrennikov, Th. M. Nieuwenhuizen, AIP, Melville, NY, 198-210, arXiv: quant-ph/0511144.

[12] E. Nelson, Quantum Fluctuation, Princeton Univ. Press, Princeton, NJ, 1985.

[13] M. Davidson, J. Math. Phys., 20:9 (1865-1869).

[14] M. Davidson, "Stochastic models of quantum mechanics - a perspective", Foundations of Probability and Physics - 4, Proceedings of the International Conference (Växjö, Sweden, June 4-9, 2006), AIP Conference Proceedings, 889, eds. G. Adenier, A. Yu. Khrennikov, C. A. Fuchs, AIP, Melville, NY, 2007, 106-119, arXiv: quant-ph/0610046.

[15] A. Bach, J. Math. Phys., 14:1 (1981), 125-132.

[16] A. Bach, Phys. Lett. A, 73:4 (1979), 287-288. 
[17] V. I. Man'ko, J. Russian Laser Research, 17:6 (1996), 579-584.

[18] V. I. Man'ko, E. V. Shchukin, J. Russian Laser Research, 22:6 (2001), 545-560.

[19] Ю.М. Белоусов, В.И. Манько, Матрица плотности. Представления и применения в статистической физике, МФТИ, М., 2004.

[20] M. A. Manko, V. I. Manko, R. V. Mendes, J. Russian Laser Research, 27:6 (2006), 507-532.

[21] S. De Nicola, R. Fedele, M. A. Manko, V. I. Manko, J. Russian Laser Research, 25:1 (2004), $1-29$.

[22] G. 't Hooft, Quantum mechanics and determinism, arXiv: hep-th/0105105.

[23] G. 't Hooft, The free-will postulate in quantum mechanics, arXiv: quant-ph/0701097.

[24] H.-T. Elze, J. Phys. Conf. Ser., 174:1 (2009), 012009, 10 pp., arXiv: 0906.1101.

[25] H.-T. Elze, J. Phys. Conf. Ser., 67:1 (2007), 012016, 11 pp., arXiv: 0704.2559.

[26] V. V. Kisil, Europhys. Lett., 72:6 (2005), 873-879, arXiv: quant-ph/0506122.

[27] А. Эйнштейн, Л. Инфельд, Эволючия физики. Развитие идей от первоначальных понятий до теории относительности и квантов, Наука, М., 1965.

[28] A. Khrennikov, J. Phys. A, 38:41 (2005), 9051-9073, arXiv: quant-ph/0505228.

[29] A. Khrennikov, Found. Phys. Lett., 18:7 (2005), 637-650.

[30] A. Khrennikov, Phys. Lett. A, 357:3 (2006), 171-176, arXiv: quant-ph/0602210.

[31] A. Khrennikov, Found. Phys. Lett., 19:4 (2006), 299-319.

[32] A. Khrennikov, Nuovo Cimento B, 121:5 (2006), 505-521, arXiv: hep-th/0604163.

[33] A. Khrennikov, Physica E, 42:3 (2010), 287-292.

[34] А. Ю. Хренников, ТМФ, 164:3 (2010), 386-393.

[35] A. Khrennikov, Nuovo Cimento B, 121:9 (2006), 1005-1021.

[36] M. Ohya, N. Watanabe, Japan. J. Appl. Math., 3:1 (1986), 197-206.

[37] A. Khrennikov, J. Modern Opt., 55:14 (2008), 2257-2267.

[38] A. Khrennikov, Europhys. Lett., 88:4 (2009), 40005, 6 pp.

[39] A. Khrennikov, Europhys. Lett., 90:4 (2010), 40004.

[40] A. Khrennikov, J. Russian Laser Research, 31:2 (2010), 191-200.

[41] I. V. Volovich, Photon antibunching, sub-Poisson statistics and Cauchy-Bunyakovsky and Bell's inequalities, arXiv: 1106.1892.

[42] I. Volovich, "Quantum cryptography in space and Bell's theorem", Foundations of Probability and Physics, Proceedings of the conference (Växjö, November 25 -December 1, 2000), Quantum Probability and White Noise Analysis, 13, ed. A. Khrennikov, World Scientific, River Edge, NJ, 2001, 364-372.

[43] M. Ohya, I. Volovich, Mathematical Foundations of Quantum Information and Computation and its Applications to Nano- and Bio-systems, Springer, New York, 2011.

[44] A. Khrennikov, Contextual Approach to Quantum Formalism, Fundamental Theories of Physics, 160, Springer, Dordrecht, 2009.

[45] A. Khrennikov, Born's rule from measurements of classical signals by threshold detectors which are properly calibrated, arXiv: 1105.4269.

[46] A. Khrennikov, M. Ohya, N. Watanabe, J. Russian Laser Research, 31:5 (2010), 462-468.

[47] Ю. И. Ожигов, Микроэлектроника, 35:1 (2006), 44-56.

[48] Y.I. Ozhigov, Dynamical diffusion as the approximation of one quantum particle dynamics, arXiv: quant-ph/0702237.

[49] Y.I. Ozhigov, Simulation of quantum dynamics via classical collective behavior, arXiv: quant-ph/0602155.

[50] Дж. фон Нейман, Математические основы квантовой механики, Наука, М., 1964.

Поступила в редакцию 21.07.2011, после доработки 10.10.2011 